\title{
The Impact of Obesity on Back and Core Muscular Endurance in Firefighters
}

\author{
John M. Mayer, ${ }^{1}$ James L. Nuzzo, ${ }^{1}$ Ren Chen, ${ }^{1}$ William S. Quillen,, ${ }^{1}$ Joe L. Verna, ${ }^{2}$ \\ Rebecca Miro, ${ }^{1}$ and Simon Dagenais ${ }^{3}$ \\ ${ }^{1}$ School of Physical Therapy \& Rehabilitation Sciences and Office of Clinical Research (RC), Morsani College of Medicine, \\ University of South Florida, 12901 Bruce B. Downs Boulevard, MDC77, Tampa, FL 33612, USA \\ ${ }^{2}$ Vert Mooney Research Foundation, San Diego, CA 92123, USA \\ ${ }^{3}$ Palladian Health, West Seneca, NY 14224, USA \\ Correspondence should be addressed to John M. Mayer, lincolnchair@health.usf.edu
}

Received 13 September 2012; Revised 25 October 2012; Accepted 25 October 2012

Academic Editor: Jack A. Yanovski

Copyright (C) 2012 John M. Mayer et al. This is an open access article distributed under the Creative Commons Attribution License, which permits unrestricted use, distribution, and reproduction in any medium, provided the original work is properly cited.

\begin{abstract}
The purpose of this study was to assess the relationships between obesity and measures of back and core muscular endurance in firefighters. Methods. A cross-sectional study was conducted in career firefighters without low back pain. Obesity measures included body mass index (BMI) and body fat percentage assessed with air displacement plethysmography. Muscular endurance was assessed with the Modified Biering Sorensen (back) and Plank (core) tests. Relationships were explored using $t$-tests and regression analyses. Results. Of the 83 participants enrolled, 24 (29\%) were obese (BMI $\geq 30)$. Back and core muscular endurance was $27 \%$ lower for obese participants. Significant negative correlations were observed for BMI and body fat percentage with back and core endurance $(r=-0.42$ to -0.52$)$. Stepwise regression models including one obesity measure (BMI, body fat percentage, and fat mass/fat-free mass), along with age and self-reported physical exercise, accounted for 17-19\% of the variance in back muscular endurance and $29-37 \%$ of the variance in core muscular endurance. Conclusions. Obesity is associated with reduced back and core muscular endurance in firefighters, which may increase the risk of musculoskeletal injuries. Obesity should be considered along with back and core muscular endurance when designing exercise programs for back pain prevention in firefighters.
\end{abstract}

\section{Introduction}

Firefighting is one of the most hazardous, physically demanding, and psychologically stressful occupations [1]. It is therefore not surprising that low back pain and related musculoskeletal injuries are very common in firefighters and the leading cause of early retirement $[1,2]$. Obesity affects more than one-third of career firefighters $[3,4]$ and has been linked with an increased risk of low back pain in the general population [5]. In the general population, obesity has also been associated with poor performance on physical fitness tests, such as back muscular endurance $[6,7]$, which has been reported to be predictive of future incidence of low back pain [8]. In the general population, poorly conditioned back and core muscles have been associated with low back pain, as individuals with low back pain have reduced strength, endurance, atrophy, fatty infiltration, and abnormal activity in various back and core muscles [8-11].

The Fire Service Joint Labor Management WellnessFitness Initiative of the International Association of Fire Fighters and International Association of Fire Chiefs recommends screening for obesity and the assessment of musculoskeletal fitness, including muscular endurance [1]. However, the relationships between obesity, low back pain, and back muscular endurance have not been assessed in firefighters. Given the link between back muscular endurance, obesity, and low back pain in the general population, along with the high incidence of low back pain in firefighters, exploring the impact of obesity on back muscular endurance in firefighters is warranted. The purpose of this study was to assess the relationships between obesity and measures of back and core muscular endurance in firefighters. 


\section{Materials and Methods}

2.1. Study Design. A cross-sectional study was conducted with firefighters from a municipal fire department in the United States. Data for this study were obtained from baseline assessments within a randomized controlled trial of progressive resistance exercise training for the low back (Grant no. EMW-2009-FP-00418, Federal Emergency Management Agency, United States Department of Homeland Security).

2.2. Participants. Male career firefighters $(n=83)$ were recruited from the entire population of firefighters $(n=573)$ of Tampa Fire Rescue (Tampa, FL, United States). The study sample consisted of 10 captains, 5 lieutenants, 13 driverengineers, 8 paramedics, and 47 firefighters without additional rank. Participants were recruited by presentations, email notices, posted information, and word-of-mouth. The University of South Florida Institutional Review Board approved the experimental protocol and all participants provided informed consent.

2.3. Screening. Candidates with interest in the study underwent telephone screening that included questions related to work status and health history, and those deemed eligible were invited to our laboratory for additional screening. On-site screening included various self-reported health history questionnaires and a physical examination. Inclusion criteria for participation were being 18 years of age or older and an active, full-duty firefighter. Candidates with cardiovascular or orthopedic contraindications to exercise, history of systemic inflammatory disease or spinal surgery, clinically meaningful current low back pain or disability, inability to participate in physical activity, presence of red flags for potential serious condition related to low back pain, abnormal resting blood pressure or heart rate, currently receiving care for spinal pain disorder/injury, currently diagnosed with or receiving care for a psychological or psychiatric disorder, currently performing progressive resistance exercises for the low back or core muscles, active workers' compensation or personal injury case, simultaneous enrollment in another clinical trial, drug or alcohol abuse within the past year, or any other condition that would put the candidate at increased safety risk or otherwise make the candidate unsuitable for this study were excluded.

2.4. Self-Reported Physical Exercise. Self-reported physical exercise was assessed by the question, "Over the past three months, on average, how many days per week have you performed any physical exercise?" This question was adapted from a specific item described in the minimum data set of the Fire Service Joint Labor Management Wellness-Fitness Initiative guidelines [1].

2.5. Body Mass Index and Body Fat Percentage. Body mass $(\mathrm{kg})$, measured with a calibrated electronic scale (Life Measurement, Inc., Concord, CA, United States), and body height $(\mathrm{cm})$, measured with a stadiometer (Novel Products Inc., Rockton, Illinois, United States), were used to calculate BMI $\left(\right.$ BMI $\left(\mathrm{kg} / \mathrm{m}^{2}\right)=$ body mass $(\mathrm{kg}) /$ body height $\left.(\mathrm{m})^{2}\right)[1]$.

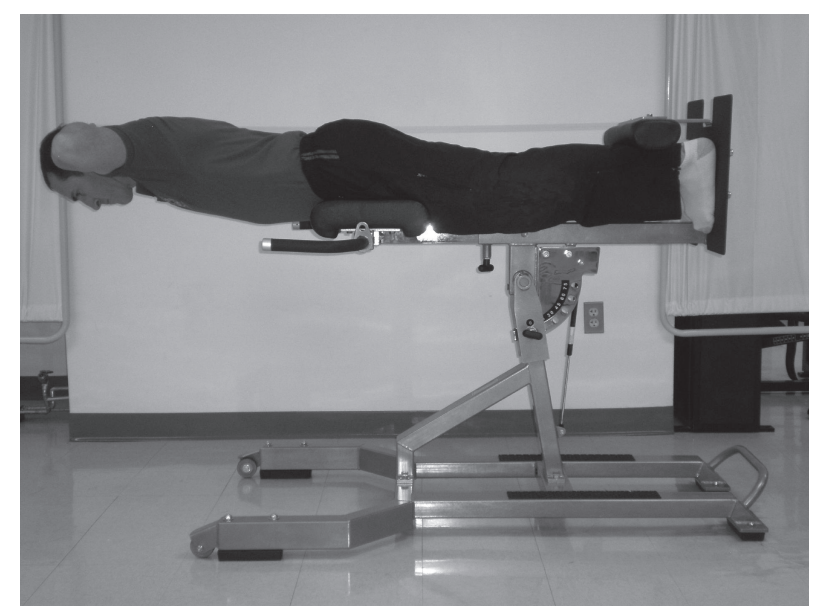

Figure 1: Depiction of the Modified Biering Sorensen tTest used to assess back muscular endurance.

Body fat percentage was assessed using whole-body air displacement plethysmography (Bod Pod, Life Measurement, Inc., Concord, CA, United States) with estimated thoracic gas volume according to the manufacturer's guidelines. For this assessment, participants wore a swim cap and underwear or compression shorts. All other clothing and jewelry were removed. The validity of air displacement plethysmography for measuring body fat percentage has been established [12]. Air displacement plethysmography, when compared with both hydrostatic weighing and dual-energy X-ray absorptiometry, agrees within $1 \%$ body fat when the average of multiple study means is calculated [12]. Also, the correlations between air displacement plethysmography and both hydrostatic weighing and dual-energy X-ray absorptiometry are strong across multiple studies $\left(R^{2}=0.78-0.94\right)[12]$.

2.6. Back and Core Muscular Endurance. Isometric back extension muscular endurance was assessed with the Modified Biering-Sorensen test [13]. For the Modified BieringSorensen test, the participant was positioned on a variable angle Roman chair (Conner Athletic Products, Jefferson, IA, United States) at the horizontal position, with the anterior superior iliac spines aligned to the superior edge of the pelvic restraint pad of the machine. The hands were placed behind the head, ankles were positioned under the ankle pad, and legs were held as straight as possible. The participant was then instructed to elevate the torso to a horizontal position (Figure 1). As soon as the horizontal position was attained, the examiner began timing with a stopwatch. The participant was verbally encouraged by the examiner to hold the position as long as possible. The participant was not informed of time elapsed while the test was being performed. If the participant's torso dropped $10^{\circ}$ below the horizontal position, the participant would be given a maximum of two warnings to reestablish the position. If the participant was unable to do so, the examiner stopped the test, and time was recorded in seconds. The Modified Biering-Sorensen test has been shown to be a reliable measure of isometric back extension muscular endurance [13]. 


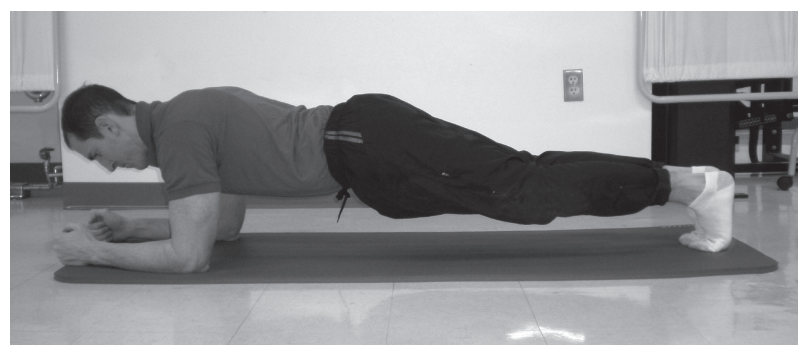

Figure 2: Depiction of the Plank test used to assess core muscular endurance.

After a four-minute rest, isometric core muscular endurance was assessed with the Plank test (Figure 2) [1]. For the Plank test, the participant was positioned as follows: prone position on a floor mat; upper body elevated and supported by the elbows; hips and legs elevated off the floor so that the neck, trunk, and lower extremities aligned in the sagittal plane (straight body alignment maintained from shoulder through hip, knee, and ankle); body supported on forearms and toes; elbows directly under the shoulders; ankles maintained at $90^{\circ}$; scapulae stabilized with elbows at $90^{\circ}$; spine in a neutral position throughout the assessment. As soon as the participant lifted the torso off the mat, a test examiner began timing with a stopwatch. The participant was verbally encouraged by the test examiner to hold the test position as long as possible. The participant was not informed of elapsed time while the test was being performed. If the participant was unable to maintain the test position, the participant would be given a maximum of two warnings from the examiner to reestablish the position. If the participant was unable to do so, the examiner stopped the test, and time was recorded in seconds. The Plank test has been shown to be a reliable measure of isometric core muscular endurance [14].

2.7. Statistical Analyses. Data were analyzed using SAS (Statistical Analysis System, version 9.2, Cary, NC, United States) and SPSS version 20 (IBM Corporation, Armonk, NY, United States). Descriptive statistics for continuous data were summarized as mean \pm standard deviation. Statistical significance was set at alpha $=0.05$. Several analyses were conducted to assess the impact of obesity on back and core muscular endurance. First, differences between the nonobese and obese participants on various measures were assessed with independent $t$-tests. Participants were categorized into one of two groups based on BMI values: nonobese-BMI < $30 \mathrm{~kg} / \mathrm{m}^{2}$, obese-BMI $\geq 30 \mathrm{~kg} / \mathrm{m}^{2}$. Due to differences in age between the groups, age was included as a covariate in the models for back and core muscular endurance comparing obese with nonobese participants. Additionally, associations between dependent variables (back and core muscular endurance) and independent variables (age, self-reported amount of any physical exercise per week, BMI, body fat percentage, fat mass, and fat-free mass) were assessed by calculating Pearson correlation coefficients for bivariable comparisons. Lastly, three separate multivariable stepwise regression analyses were conducted for both back and core endurance, each using one of three measures for obesity (BMI, body fat percentage, or fat mass/fat-free mass) along with age and self-reported physical exercise as independent variables. Multiple measures of obesity were selected because of the potentially different application for each measure. For example, BMI is an indirect measure of body composition that is frequently used as an efficient test in occupational, health, and fitness settings. Body fat percentage, fat mass, and fat-free mass provide more direct markers of body composition, yet require specialized testing equipment. Multiple measures of obesity were not included within the same stepwise regression model because the bivariable correlations among these measures were moderate to strong, ranging from $r=0.45$ to $r=0.96$ (Table 2). Due to the low bivariable correlations among the included independent variables within each of the multivariable stepwise regression analyses, collinearity was not deemed an issue and, therefore, variance inflation factor was not considered.

In addition to the analyses used to assess the impact of obesity on muscular endurance, the rate at which BMI misclassified subjects as obese or nonobese was also assessed by calculating false negative and false positive rates. Misclassifications of obesity were based on standard body fat percentage criteria for defining obesity (obese $\geq 25 \%$ body fat; not obese $<25 \%$ body fat) [15].

\section{Results}

Age, physical exercise, obesity, and muscular endurance data of the participants stratified by obesity category according to BMI are summarized in Table 1. The mean BMI of all the firefighters was $28.1 \mathrm{~kg} / \mathrm{m}^{2}$, which is in the overweight range. According to standard BMI categories [1], 21.7\% ( $n=18$ ) of the participants had a normal BMI, $49.4 \%$ ( $n=41)$ were overweight, and 28.9\% $(n=24)$ were obese. Based on body fat percentage [15], 57.6\% of the participants were nonobese and $42.4 \%$ of participants were obese. Obese firefighters according to BMI categories had poorer back and core muscular endurance times compared with the nonobese firefighters. Of the 83 participants, 19 (22.9\%) were misclassified by BMI according to body fat percentage criteria. A total of $15(42.9 \%)$ participants were misclassified by BMI as nonobese when they were actually obese (i.e., falsenegative misclassifications). A total of 4 (8.3\%) participants were misclassified by BMI as obese when they were actually nonobese (i.e., false-positive misclassifications).

Bivariable Pearson's correlation coefficients $(r)$ comparing age, physical exercise, BMI, body fat percentage, fat mass, fat-free mass, and muscular endurance data are presented in Table 2. For back muscular endurance, significant negative correlations were noted with age, BMI, body fat percentage, and fat mass. For core muscular endurance, significant negative correlations were noted with BMI, body fat percentage, fat mass, and fat-free mass.

For back muscular endurance, the obesity measure was the only significant independent variable in the three stepwise multivariable regression models, which accounted for $16-19 \%$ of the variance in back muscular endurance 
TABLe 1: Age, physical exercise, obesity, and muscular endurance data of participants stratified by obesity categories.

\begin{tabular}{|c|c|c|c|c|}
\hline & Total $(n=83)$ & Non-obese $\left(\mathrm{BMI}<30 \mathrm{~kg} / \mathrm{m}^{2}\right)(n=59)$ & Obese $\left(\mathrm{BMI} \geq 30 \mathrm{~kg} / \mathrm{m}^{2}\right)(n=24)$ & $P$ value \\
\hline Age $(y)$ & $35.7 \pm 9.4$ & $34.1 \pm 9.6$ & $39.7 \pm 7.7$ & 0.013 \\
\hline Years as career firefighter & $9.7 \pm 8.9$ & $9.1 \pm 9.0$ & $11.2 \pm 8.8$ & 0.341 \\
\hline Any exercise $(\mathrm{d} / \mathrm{wk})$ & $3.2 \pm 1.3$ & $3.3 \pm 1.3$ & $2.9 \pm 1.2$ & 0.139 \\
\hline Body height (cm) & $178.2 \pm 7.7$ & $177.4 \pm 7.0$ & $180.2 \pm 9.0$ & 0.130 \\
\hline Body mass (kg) & $89.5 \pm 14.8$ & $82.9 \pm 9.6$ & $105.7 \pm 12.7$ & $<0.001$ \\
\hline BMI $\left(\mathrm{kg} / \mathrm{m}^{2}\right)$ & $28.1 \pm 3.7$ & $26.3 \pm 2.4$ & $32.5 \pm 2.3$ & $<0.001$ \\
\hline Body fat (\%) & $22.5 \pm 8.3$ & $19.5 \pm 7.2$ & $29.8 \pm 6.0$ & $<0.001$ \\
\hline Fat mass $(\mathrm{kg})$ & $20.9 \pm 10.0$ & $16.6 \pm 7.3$ & $31.6 \pm 7.2$ & $<0.001$ \\
\hline Fat-free mass (kg) & $68.6 \pm 8.5$ & $66.3 \pm 6.2$ & $74.2 \pm 10.7$ & 0.002 \\
\hline Back muscular endurance(s) & $90.5 \pm 26.4$ & $98.2 \pm 23.0$ & $71.3 \pm 24.6$ & $<0.001^{*}$ \\
\hline Core muscular endurance(s) & $140.2 \pm 56.4$ & $151.9 \pm 56.6$ & $111.5 \pm 45.4$ & $0.001^{*}$ \\
\hline
\end{tabular}

Values reported as raw mean \pm standard deviation. Statistical comparisons were made between the nonobese and obese groups. Any exercise: self-reported days per week of any physical over the past three months; BMI: body mass index.

${ }^{*} P$ values after covaring for age.

Table 2: Matrix of bivariable Pearson's correlation coefficient $(r)$ values between selected age, physical exercise, obesity, and muscular endurance data.

\begin{tabular}{|c|c|c|c|c|c|c|c|}
\hline & Core endurance & Age & Any exercise & BMI & Body Fat \% & Fat mass & Fat-free mass \\
\hline Back endurance & $0.36^{\mathrm{b}}$ & $-0.22^{\mathrm{a}}$ & NS & $-0.44^{\mathrm{b}}$ & $-0.45^{\mathrm{b}}$ & $-0.46^{\mathrm{b}}$ & -0.15 \\
\hline Core endurance & 1.00 & NS & NS & $-0.47^{\mathrm{b}}$ & $-0.52^{\mathrm{b}}$ & $-0.55^{\mathrm{b}}$ & $-0.26^{\mathrm{a}}$ \\
\hline Age & & 1.00 & NS & $0.34^{\mathrm{b}}$ & $0.37^{\mathrm{b}}$ & $0.32^{\mathrm{b}}$ & -0.06 \\
\hline Any exercise & & & 1.00 & $-0.25^{\mathrm{a}}$ & $-0.33^{\mathrm{b}}$ & $-0.28^{\mathrm{b}}$ & 0.09 \\
\hline BMI & & & & 1.00 & $0.77^{\mathrm{b}}$ & $0.85^{\mathrm{b}}$ & $0.45^{\mathrm{b}}$ \\
\hline Body fat \% & & & & & 1.00 & $0.96^{\mathrm{b}}$ & 0.02 \\
\hline Fat mass & & & & & & 1.00 & $0.28^{\mathrm{a}}$ \\
\hline Fat-free mass & & & & & & & 1.00 \\
\hline
\end{tabular}

Key: ${ }^{\mathrm{a}} P \leq 0.05{ }^{\mathrm{b}} \mathrm{P} \leq 0.01$.

Any exercise: self-reported days per week of any physical exercise over the past three months; back endurance: back muscular endurance assessed with the modified Biering Sorensen test(s); core endurance: core muscular endurance assessed with the Plank test(s); BMI: body mass index; NS: not significant.

TABLE 3: Multivariable stepwise regression models for back muscular endurance displaying significant independent variables.

\begin{tabular}{|c|c|c|c|c|c|}
\hline Label & Coefficient & SE & $P$ & Partial $r^{2}$ & Model $r^{2}$ \\
\hline \multicolumn{6}{|l|}{ Model 1: body mass index } \\
\hline Intercept & 173.44 & 19.21 & $<0.001$ & & \\
\hline Body mass index $\left(\mathrm{kg} / \mathrm{m}^{2}\right)$ & -2.92 & 0.68 & $<0.001$ & 0.19 & 0.19 \\
\hline \multicolumn{6}{|l|}{ Model 2: body fat $\%$} \\
\hline Intercept & 118.49 & 7.48 & $<0.001$ & & \\
\hline Body fat $(\%)$ & -1.22 & 0.32 & $<0.001$ & 0.16 & 0.16 \\
\hline \multicolumn{6}{|c|}{ Model 3: fat mass and fat-free mass } \\
\hline Intercept & 113.08 & 5.95 & $<0.001$ & & \\
\hline Fat mass (kg) & -1.05 & 0.26 & $<0.001$ & 0.17 & 0.17 \\
\hline
\end{tabular}

SE: standard error.

(Table 3). With a 1-point increase in BMI, back muscular endurance decreases by 2.9 seconds. With a 1-point increase in body fat percentage, back muscular endurance decreases by 1.2 seconds. With a 1-point increase in fat mass, back muscular endurance decreases by 1.1 seconds. For core muscular endurance, the obesity measure and age were significant independent variables in all three of the stepwise multivariable regression models, which accounted for 29$37 \%$ of the variance in core muscular endurance (Table 4). With a 1-point increase in BMI, core muscular endurance decreases by 8.6 seconds. With a 1-point increase in body fat percentage, core muscular endurance decreases by 4.4 seconds. With a 1-point increase in fat mass, core muscular endurance decreases by 3.7 seconds. 
TABLE 4: Multivariable stepwise regression models for core muscular endurance displaying significant independent variables.

\begin{tabular}{lcccc}
\hline Label & Coefficient & SE & $P$ & Partial $r^{2}$ \\
\hline Model 1: body mass index & & & & \\
$\quad$ Intercept & 324.49 & 41.92 & $<0.001$ & 0.22 \\
Body mass index $\left(\mathrm{kg} / \mathrm{m}^{2}\right)$ & -8.62 & 1.55 & $<0.001$ & 0.07 \\
$\quad$ Age (y) & 1.64 & 0.61 & 0.009 & 0.29 \\
\hline Model 2: body fat \% & & & 0.27 \\
$\quad$ Intercept & 173.22 & 21.68 & $<0.001$ & 0.08 \\
Body fat (\%) & -4.36 & 0.68 & $<0.001$ & 0.27 \\
Age (y) & 1.81 & 0.58 & 0.003 & 0.34 \\
\hline Model 3: fat mass and fat-free mass & & & \\
Intercept & 158.42 & 20.41 & $<0.001$ & 0.31 \\
Fat mass (kg) & -3.71 & 0.55 & $<0.001$ & 0.07 \\
Age (y) & 1.65 & 0.56 & 0.004 & 0.31 \\
\hline
\end{tabular}

SE: standard error.

\section{Discussion}

The findings of this study indicate that obesity has a significant negative impact on back and core muscular endurance in firefighters, regardless of which measure of obesity is assessed. Similar trends were observed for the relationships between all markers of obesity (BMI, body fat percentage, or fat mass/fat-free body mass) assessed in this study and back and core muscular endurance.

Several factors may explain the poorer back and muscular endurance times in obese firefighters. First, the biomechanical strategies required for the muscular endurance tests used in this study are likely impacted by body mass. The Modified Biering Sorensen and the Plank tests depend on gravity's action on body mass for the entire load and the ability to support body mass against gravity for an extended period of time. Thus, individuals with high BMI values are likely at a biomechanical disadvantage during these tests given the higher body loads that need to be supported against gravity. It is unclear if the same relationship between BMI and back and core muscular endurance exists when testing strategies are used that are not dependent on gravity's effect on body mass and exercise load. The lack of significant relationships between fat-free mass and muscular endurance measures in the bivariable and multivariable regression models may indicate that overall body mass may play a larger role than muscle mass in back and core muscular endurance performance testing. Other conceivable explanations for the poorer muscular endurance times in obese firefighters include possible differences between obese and nonobese individuals in physiology and morphology of the back and core muscles, general fitness levels, perceived exertion levels on fitness tests, and familiarity with physical fitness exercise, along with a possible negative influence of other comorbidities associated with obesity.

Considering that the multivariable regression models for back and core muscular endurance accounted for only 16$19 \%$ and $29-37 \%$ of the variance, respectively, these models do not account for a large portion of the variance in back and core muscular endurance. Therefore, other factors that were not measured in this study may be involved in performance on the muscular endurance tests. Also, because the total portion of variance accounted for in the models was low, back and core muscular endurance should be directly assessed and should not be predicted from age, self-reported amount of any physical exercise per week, and measures of obesity.

The present study's findings on the relationship of body composition and back muscular endurance in firefighters appear to be similar to the relationships observed in the general population. We uncovered four previous studies that assessed relationships between back muscular endurance (as assessed by some version of the Biering Sorensen test) and body composition in the general population $[6,7,16,17]$. In three of these four studies $[6,7,16]$, significant moderate negative correlations between measures of body composition (BMI and body fat percentage) and back muscular endurance were observed.

In the present study, BMI and body fat percentage were elevated in a large proportion of participants, indicating a high prevalence of overweight or obese firefighters in this study. In the current study, 29\% of the firefighters were obese according to BMI, and $42 \%$ of firefighters were obese according to body fat percentage. These findings are consistent with previous studies of obesity in firefighters which reported that approximately $34 \%$ of firefighters are obese according to BMI $[3,4]$ and approximately $48 \%$ are obese according to body fat percentage [3]. In the present study, BMI misclassified $42.9 \%$ of obese firefighters (according to body fat percentage criteria defining obese as $\geq 25 \%$ ) as nonobese (i.e., false negative diagnosis). Such misclassifications appear to be common in male firefighters, as a previous study reported a false negative rate of $32.9 \%$ [3]. Given the relatively high rate of mismatch between BMI and body fat percentage, assessing both BMI and body fat percentage is reasonable since each method may give unique information on the health status of firefighters.

In addition to the relationship between obesity and poor back and core muscular endurance observed in the present study, obesity has been linked with decreased job performance and increased risk of disability in firefighters. 
A recent study assessed fitness parameters among firefighters, including BMI, body fat percentage, and performance on a physical ability test that consisted of climbing stairs, rolling, lifting, pulling, threading a hose coupling to a hydrant, and advancing a hose, wielding a sledgehammer, and rescuing a mannequin [18]. In this study, poor performance on the physical ability test was correlated with high BMI $(r=0.34$, $P<0.01)$ and high body fat percentage $(r=0.36, P<0.01)$. A longitudinal study of firefighters reported that obesity was a significant risk factor for job disability, and that every unit increase in BMI was associated with a 5\% increase in risk of job disability [4]. This study also reported that obese firefighters $(\mathrm{BMI} \geq 30)$ had a hazard ratio of 2 for an adverse employment event, defined as injured-duty status, termination of duty, resignation, premature retirement, or death, when compared to those with a BMI $<27$. Whether obesity is associated with increased risk for musculoskeletal disorders, such as low back pain, in firefighters is unknown and requires further study.

The present study has noteworthy limitations that should be considered when interpreting its findings. First, this study enrolled a relatively small number of participants, which limited the number of independent variables that could be considered in multivariable regression models. The study sample was powered based on potential differences in outcomes of the randomized trial, not the regression models of this cross-sectional analysis. Also, the present study only enrolled male participants from a single fire department in the southeastern United States, and it is unclear if results can be generalized to firefighters in other settings. The crosssectional, observational design used in this study can only report on associations observed at the time of measurement and is therefore unable to establish causation between back and core muscular endurance and body composition. Additionally, it is unclear if performance on the back and core muscular endurance tests reflects the ability to perform physical activities required of firefighters. Although physical factors such as back and core muscular endurance and obesity measures are associated with low back pain, other factors that are associated with low back pain, such as psychosocial occupational factors, were not measured in this study. Finally, the measure of self-reported physical activity used in this study, although a part of the minimum data set established by the Fire Service Joint Labor Management Wellness-Fitness Initiative, has yet to be validated.

\section{Conclusions}

Obesity as measured by increased BMI and body fat percentage is associated with decreased back and core muscular endurance in firefighters, which is consistent with findings in the general population. Given the relationship between increased incidence of low back pain in obese individuals and individuals with poor back muscular endurance, exercise programming for back injury prevention in firefighters should address improving both body composition and muscular endurance. Additional research is needed to assess the impact of exercise training and improving body composition on increasing back and core muscular endurance, improving job performance, and reducing risk for low back pain in firefighters.

\section{Disclosure}

J. L. Verna is the patent holder of the exercise testing device used to assess back muscular endurance in this study.

\section{Conflict of Interests}

The authors declare that they have no conflict of interests.

\section{Acknowledgments}

This study was funded by a Fire Prevention and Safety Grant (no.: EMW-2009-FP-00418) from the Federal Emergency Management Agency, U.S. Department of Homeland Security. The authors wish to thank Tampa Fire Rescue for its assistance in organizing and implementing this study.

\section{References}

[1] International Association of Fire Fighters and International Association of Fire Chiefs, The Fire Service Joint Labor Management Wellness-Fitness Initiative, International Association of Fire Fighters and International Association of Fire Chiefs, Spokane Valley, Wash, USA, 3rd edition edition, 2008.

[2] G. S. Poplin, R. B. Harris, K. M. Pollack, W. F. Peate, and J. L. Burgess, "Beyond the fireground: injuries in the fire service," Injury Prevention, vol. 18, no. 4, pp. 228-233, 2011.

[3] W. S. C. Poston, C. K. Haddock, S. A. Jahnke, N. Jitnarin, B. C. Tuley, and S. N. Kales, "The prevalence of overweight, obesity, and substandard fitness in a population-based firefighter cohort," Journal of Occupational and Environmental Medicine, vol. 53, no. 3, pp. 266-273, 2011.

[4] E. S. Soteriades, R. Hauser, I. Kawachi, D. C. Christiani, and S. N. Kales, "Obesity and risk of job disability in male firefighters," Occupational Medicine, vol. 58, no. 4, pp. 245-250, 2008.

[5] R. Shiri, J. Karppinen, P. Leino-Arjas, S. Solovieva, and E. Viikari-Juntura, "The association between obesity and low back pain: a meta-analysis," The American Journal of Epidemiology, vol. 171, no. 2, pp. 135-154, 2010.

[6] Å. Dedering, G. Németh, and K. Harms-Ringdahl, "Correlation between electromyographic spectral changes and subjective assessment of lumbar muscle fatigue in subjects without pain from the lower back," Clinical Biomechanics, vol. 14, no. 2, pp. 103-111, 1999.

[7] C. E. Mbada, O. Ayanniyi, R. A. Adedoyin, and O. E. Johnson, "Static endurance of the back extensor muscles: association between performance and reported reasons for test termination," Journal of Musculoskeletal Research, vol. 13, no. 1, pp. 13-21, 2010.

[8] C. Demoulin, M. Vanderthommen, C. Duysens, and J. M. Crielaard, "Spinal muscle evaluation using the sorensen test: a critical appraisal of the literature," Joint Bone Spine, vol. 73, no. 1, pp. 43-50, 2006.

[9] J. A. Hides, M. J. Stokes, M. Saide, G. A. Jull, and D. H. Cooper, "Evidence of lumbar multifidus muscle wasting ipsilateral to symptoms in patients with acute/subacute low back pain," Spine, vol. 19, no. 2, pp. 165-172, 1994. 
[10] V. Mooney, J. Gulick, M. Perlman et al., "Relationships between myoelectric activity, strength, and MRI of lumbar extensor muscles in back pain patients and normal subjects," Journal of Spinal Disorders, vol. 10, no. 4, pp. 348-356, 1997.

[11] G. L. Soderberg and J. O. Barr, "Muscular function in chronic low-back dysfunction," Spine, vol. 8, no. 1, pp. 79-85, 1983.

[12] D. A. Fields, M. I. Goran, and M. A. Mccrory, "Body-composition assessment via air-displacement plethysmography in adults and children: a review," The American Journal of Clinical Nutrition, vol. 75, no. 3, pp. 453-467, 2002.

[13] B. E. Udermann, J. M. Mayer, J. E. Graves, and S. R. Murray, "Quantitative assessment of lumbar paraspinal muscle endurance," Journal of Athletic Training, vol. 38, no. 3, pp. 259262, 2003.

[14] K. L. Schellenberg, J. M. Lang, K. M. Chan, and R. S. Burnham, "A clinical tool for office assessment of lumbar spine stabilization endurance: prone and supine bridge maneuvers," The American Journal of Physical Medicine and Rehabilitation, vol. 86, no. 5, pp. 380-386, 2007.

[15] D. O. Okorodudu, M. F. Jumean, V. M. Montori et al., "Diagnostic performance of body mass index to identify obesity as defined by body adiposity: a systematic review and meta-analysis," International Journal of Obesity, vol. 34, no. 5, pp. 791-799, 2010.

[16] L. E. Gibbons, T. Videman, and M. C. Battié, "Determinants of isokinetic and psychophysical lifting strength and static back muscle endurance: a study of male monozygotic twins," Spine, vol. 22, no. 24, pp. 2983-2990, 1997.

[17] M. Pääsuke, E. Johanson, M. Proosa, J. Ereline, and H. Gapeyeva, "Back extensor muscle fatigability in chronic low back pain patients and controls: relationship between electromyogram power spectrum changes and body mass index," Journal of Back and Musculoskeletal Rehabilitation, vol. 16, no. 1, pp. 17-24, 2002.

[18] M. A. Michaelides, K. M. Parpa, L. J. Henry, G. B. Thompson, and B. S. Brown, "Assessment of physical fitness aspects and their relationship to firefighters' job abilities," Journal of Strength and Conditioning Research, vol. 25, no. 4, pp. 956-965, 2011. 


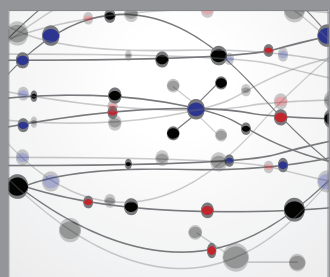

The Scientific World Journal
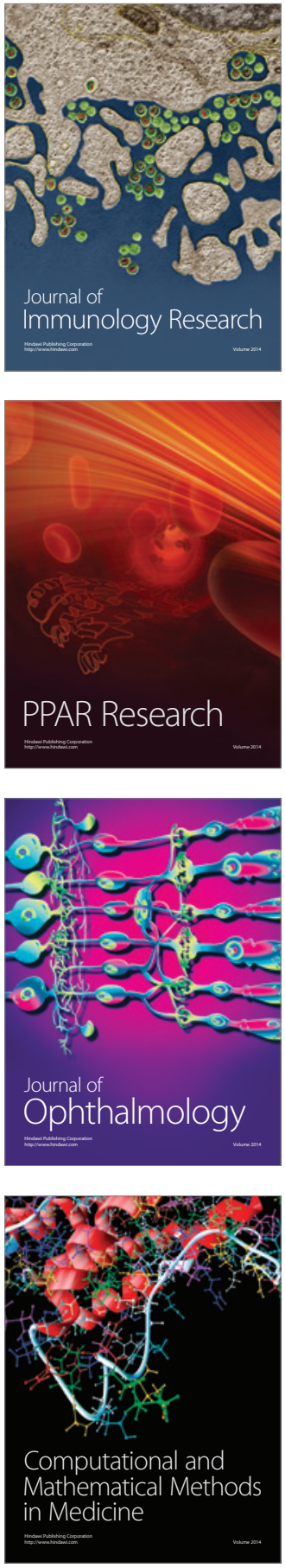

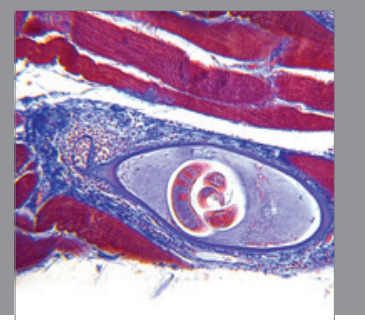

Gastroenterology

Research and Practice
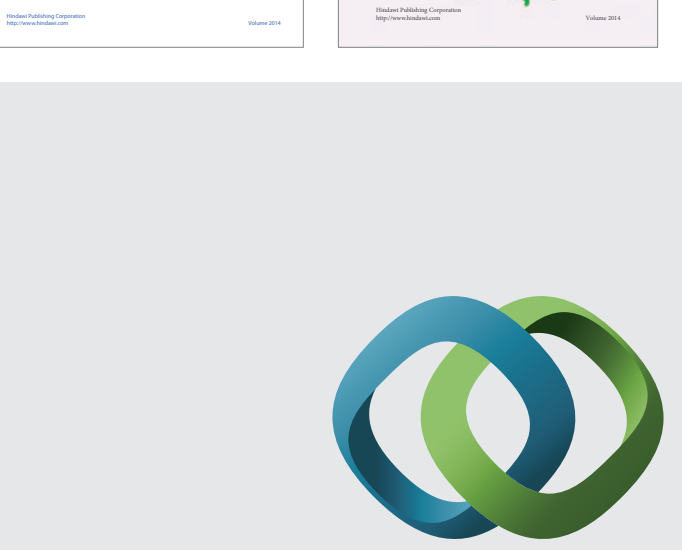

\section{Hindawi}

Submit your manuscripts at

http://www.hindawi.com
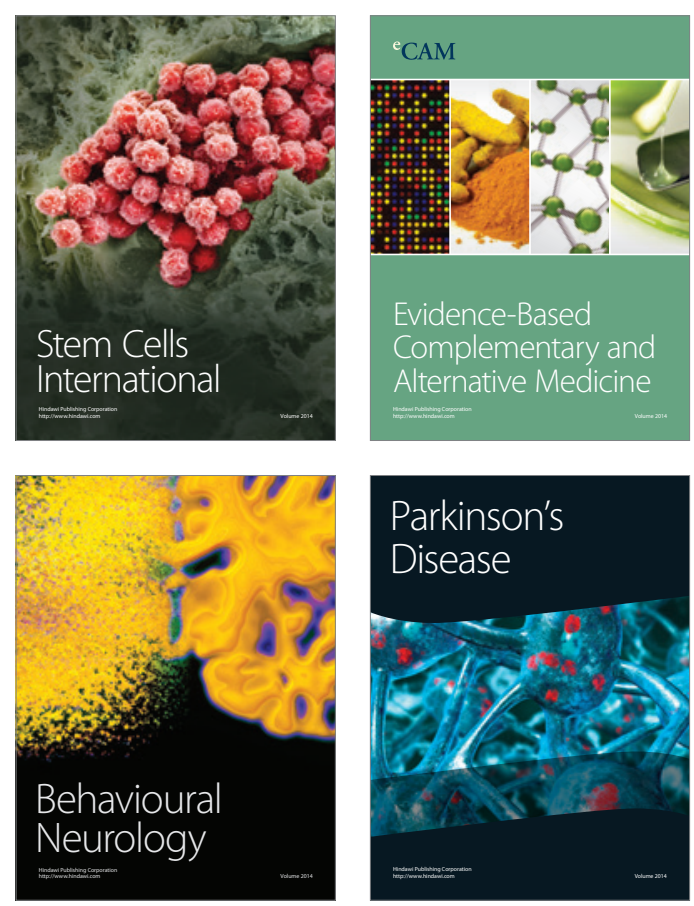

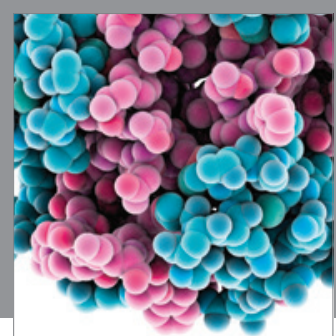

Journal of
Diabetes Research

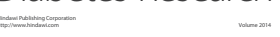

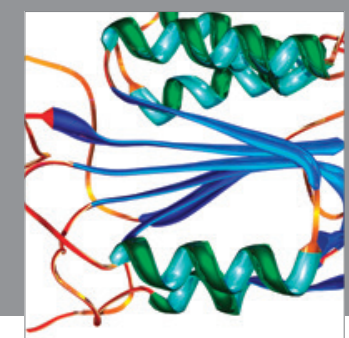

Disease Markers
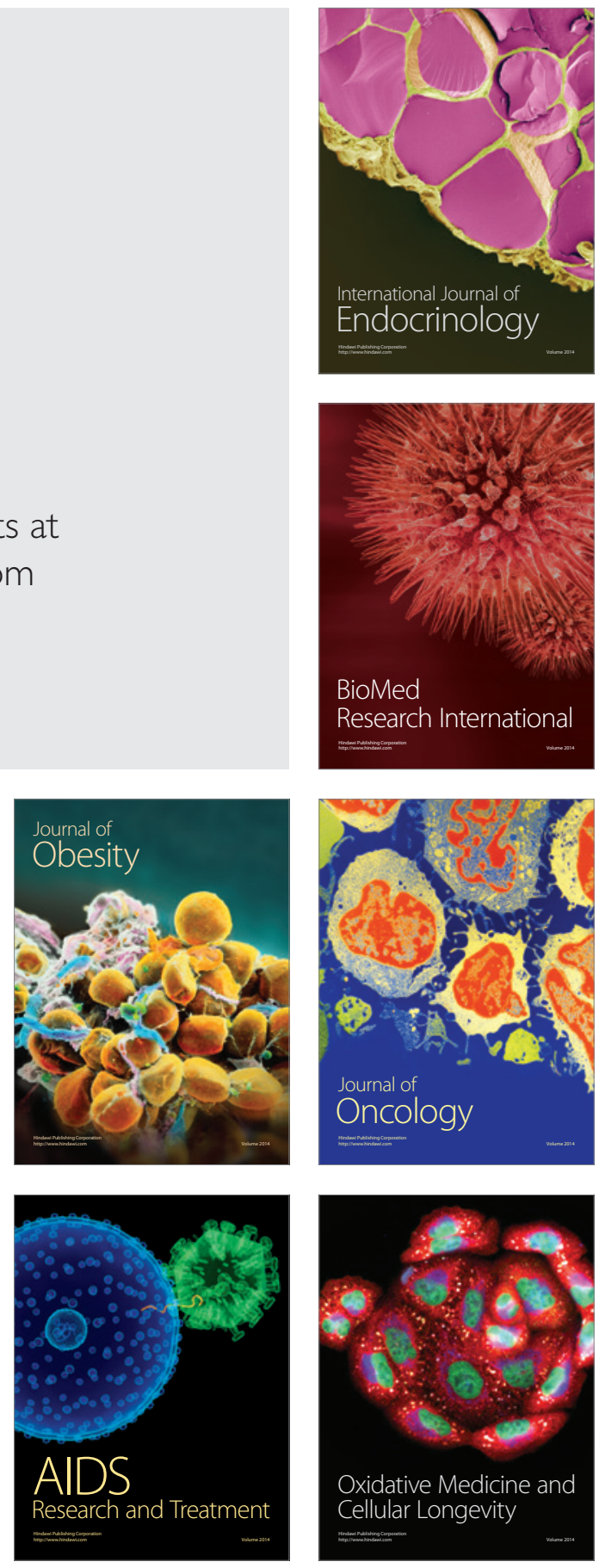